\section{Stomach Tumors Stem from Bone Marrow}

The surprising finding that gastric tumors may arise from bone marrow cells, not stomach cells, may cause researchers to rethink the origins of epithelial cancers.

There is a well-established correlation between chronic infection with the ulcercausing bacterium Helicobacter pylori and the development of gastric cancer in humans. Most researchers believe that over time, infection and inflammation cause changes in gastric epithelial cells that ultimately lead to cancer development. Noting that bone marrow-derived cells (BMDCs) migrate to areas of inflammation and attempt to repair damage, one research team recently sought to determine what role these cells play in cancer initiation and development.

In the 26 November 2004 issue of Science, JeanMarie Houghton of the University of Massachusetts Medical School (Worcester, MA), Timothy C. Wong, now at the Columbia College of Physicians and Surgery (New York, NY), and their colleagues present the results of a study in mice that challenges conventional thinking on cancer development. The group irradiated $\mathrm{C} 57 \mathrm{BL} / 6$ mice to destroy their bone marrow and then transplanted them with bone marrow cells that had been labeled with a marker molecule that could be used to differentiate between host and donor cells. They then inoculated these mice with Helicobacter felis, which causes in mice similar symptoms to those caused in humans by $H$. pylori. At 20 weeks after inoculation with $H$. felis, BMDCs had repopulated much of the stomach lining. The number of BMDCs in the stomach increased with time and eventually produced cancerous growths.

Houghton points out that BMDCs share many characteristics with cancer cells, including "the ability to avoid apoptosis, the ability to continually divide, and the ability to travel". By learning how these cells maintain these growth characteristics, researchers may be able to devise new, more effective treatments.
There is still a great deal of work to be done, however, before BMDCs can be applied to treating humans. Houghton tells Lab Animal, "The next steps are to identify which cell within the bone marrow is responsible for cancer, determine what are the local signals responsible for BMDCs homing to the area, and, more importantly, the signals (or lack of) that keep these cells in a 'stemlike' state of growth. Once we know this, we will identify ways to shut these programs down as a means of establishing effective anticancer therapy."

-Tanja Schub

\section{Bacteria-Based Allergy Therapy Helps Dogs}

A vaccine that includes a common foodborne pathogen reversed severe allergies to peanut, milk, and wheat in dogs, suggesting a possible treatment for the millions of people who suffer from food allergies.

Peanut and tree nut allergies affect approximately 3 million Americans and cause the most severe food-induced allergic reactions, including life-threatening anaphylaxis. There are no good treatments for these and other food allergies, and avoidance of the allergen is often the only way to prevent an allergic reaction in a sensitized patient.

The food-borne pathogen, Listeria monocytogenes, is a potent activator of $\mathrm{T}$ cells that has previously been shown to reduce the production of the IgE antibodies associated with allergic reactions and reverse allergeninduced airway hyperreactivity in mice. In the 12 November 2004 online edition of Allergy (doi: 10.1111/1.1398-9995.2004. 00711.x), a group led by Oscar L. Frick, Professor Emeritus of Pediatrics-AllergyImmunology at the University of California, San Francisco, reports promising results in a study of dogs with severe food allergies.

The research team used nine dogsfour with peanut allergies and five with milk and wheat allergies. They inoculated the dogs with the pertinent antigen(s) and heat-killed L. monocytogenes (HKL). Following vaccination, the dogs underwent oral challenge with the offending foods, and the researchers monitored them closely for allergic symptoms, including vomiting, lethargy, and diarrhea. Even 10 weeks after vaccination, all of the dogs were able to tolerate significantly more of the trigger food with minor or no symptoms; indeed, one of the peanut-allergic dogs was able to eat a dose of ground peanut 100 times what would have caused a reaction before vaccination.

As the authors write in Allergy, “...these studies suggest that HKL as an adjuvant might be very effective as curative immunotherapy for allergic diseases, including allergic asthma and food allergy in humans."

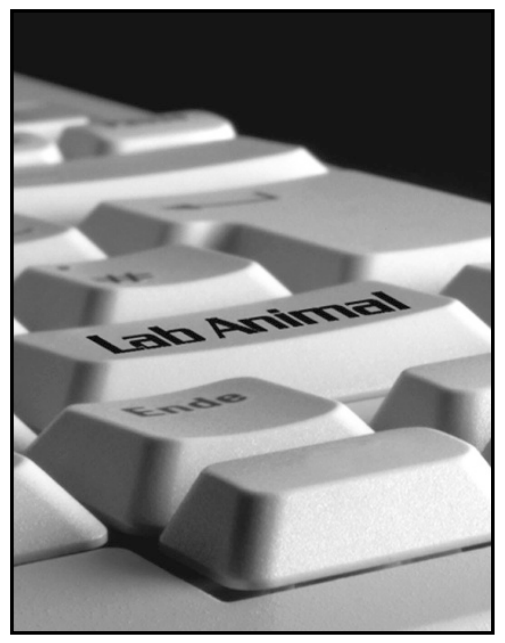

www.labanimal.com 